\title{
Interplay between magnetic anisotropies in CoAu and Co films and antidot arrays: effects on the spin configuration and hysteretic behavior.
}

\begin{tabular}{|r|l|}
\hline Journal: & Physical Chemistry Chemical Physics \\
\hline Manuscript ID & CP-ART-04-2018-002323.R2 \\
\hline Article Type: & Paper \\
\hline Date Submitted by the Author: & $17-$ May-2018 \\
\hline Complete List of Authors: & $\begin{array}{l}\text { Spizzo, Federico; Università di Ferrara, Dipartimento di Fisica e Scienze } \\
\text { della Terra } \\
\text { Del Bianco, Lucia; Universita degli Studi di Ferrara, Department of Physics } \\
\text { and Earth Sciences } \\
\text { Coisson, Marco; Istituto Nazionale di Ricerca Metrologica, Nanoscience and } \\
\text { Materials } \\
\text { Chinni, Federico; Universita degli Studi di Ferrara, Department of Physics } \\
\text { and Earth Sciences } \\
\text { Mattarello, Valentina; University of Padova, } \\
\text { maurizio, chiara; Università degli Studi di Padova, Physics and Astronomy } \\
\text { Department } \\
\text { Mattei, Giovanni; University of Padova, Dept. of Physics and Astronomy }\end{array}$ \\
\hline \hline
\end{tabular}




\title{
Interplay between magnetic anisotropies in CoAu and Co films and antidot arrays: effects on
} the spin configuration and hysteretic behavior.

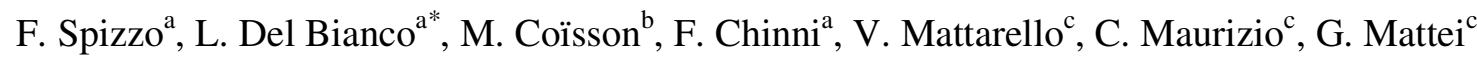 \\ ${ }^{a}$ Dipartimento di Fisica e Scienze della Terra, Università di Ferrara, I-44122 Ferrara, Italy \\ ${ }^{\mathrm{b}}$ INRIM, Nanoscience and Materials Division, I-10135 Torino, Italy \\ ${ }^{\mathrm{c}}$ Dipartimento di Fisica e Astronomia, Università di Padova, I-35131 Padova, Italy \\ *Corresponding author. E-mail address: lucia.delbianco@unife.it
}

\begin{abstract}
We studied i) a set of three Co:Au continuous films, grown by sputtering co-deposition $(\sim 80 \mathrm{~nm}$ thick) and concentration ratio 2:1, 1:1 and 1:0 (i.e., a pure Co film was also included) and ii) a corresponding set of antidot arrays, produced by nanosphere lithography with the same hexagonal pattern (nominal lattice periodicity $\sim 520 \mathrm{~nm}$ ). The samples were investigated by atomic and magnetic force microscopy and SQUID magnetometry. A twofold aim was fulfilled: to gain information on the magnetism of the CoAu compound (saturation magnetization, effective in-plane and out-of-plane anisotropy, exchange stiffness constant, magnetostrictive behavior) and to compare the magnetic behavior of the continuous and patterned samples. The continuous films exhibited a variety of hysteretic properties and magnetic configurations, ruled by the interplay between different magnetic anisotropy terms (magnetocrystalline, magnetoelastic, shape). The $\mathrm{Co}_{1} \mathrm{Au}_{1}$ film was anisotropic in the plane, whereas $\mathrm{Co}_{2} \mathrm{Au}_{1}$ and $\mathrm{Co}$ were isotropic and had an out-ofplane magnetization component; stripe domains were observed in $\mathrm{Co}_{2} \mathrm{Au}_{1}$, resulting in a transcritical hysteresis loop. A key role in determining these properties was ascribed to the magnetoelastic anisotropy term. Unlike the continuous films, the antidot arrays showed a similar hysteretic behavior and important similarities in the spin configuration were pointed out, despite the different composition. We argue, also based on micromagnetic simulations, that this occurred because the nanopatterning enabled a local modification of the shape anisotropy, thus smoothing out the differences observed in the continuous films.
\end{abstract}




\section{Introduction}

In the field of magnetic materials, the design of innovative systems and devices is inextricably linked to the possibility of assessing and tuning the magnetic anisotropy, which refers to the existence of preferred magnetization directions. ${ }^{1,2,3,4,5,6}$ The magnetic domain configuration as well as the hysteretic behavior of a magnetic system and the related properties - coercivity, remanent magnetization and squareness ratio, which ultimately determine the possible technological application - depend on the macroscopic magnetic anisotropy. In turn, in many cases, the latter arises from the complex interplay among different anisotropy contributions, the principal ones being: i) the magnetocrystalline term, related to the crystallography of the material; ii) the magnetoelastic term, originating from the coupling between mechanical stress and magnetization, and iii) the shape term, strictly connected to the magnetostatic energy of the system. ${ }^{7}$

Given a magnetic system with a fixed chemical composition, the shape anisotropy appears as the type that can be more easily tailored through a proper design of the morphological features, whereas the magnetocrystalline term can be modified, to some extent, by varying the crystallinity degree. For instance, it is known that in amorphous or nanocrystalline materials the magnetocrystalline anisotropy can be strongly reduced, compared to the coarse-grained counterparts, and even almost annihilated. ${ }^{8,9,10}$ In the case of magnetostrictive materials, magnetoelastic anisotropy may arise due to the action of an internal mechanical stress caused by the production process. For instance, this occurs in metallic glasses prepared by ultrarapid quenching ${ }^{11,12,13}$ as well as in materials subjected to mechanical milling. ${ }^{14,15}$ In thin films a mechanical stress can be produced because of the bonding with the substrate. ${ }^{16}$ Hence, the magnetoelastic anisotropy can be varied by controlling the field of internal and applied stresses acting on the system ${ }^{17,18}$, an approach that appears especially promising in the case of layered materials grown on flexible substrates. ${ }^{19} 20$

Nowadays, thanks to the impressive advances in deposition and lithography techniques, ${ }^{21}$ a control of the morphological characteristics of a magnetic system can be achieved even at the nanoscale, which meets the growing demand for the miniaturization of magnetic devices (magnetic sensors, high-density data storage media). The nanopatterning of a continuous film, namely the creation of periodic arrays of nanosized dots or antidots (holes), may alter the different anisotropy contributions and, hence, the interplay among them. A modulation of the shape anisotropy can be induced by nanopatterning ${ }^{22,23}$ and the internal stress field may be also modified, thus affecting the magnetoelastic term. Moreover, the magnetostatic interactions among the nanoelements (dots or antidots) can give rise to a configurational anisotropy, related to the symmetry of the underlying periodic lattice. $^{24,25}$ 
In short, the tailoring of size, shape and spatial density of the nanoelements enables a suitable modification of the magnetic properties of the continuous film counterpart. ${ }^{26,27,28,29,30}$ Hence, it is well demonstrated that, given a set of continuous films with the same magnetic behavior, they can be magnetically diversified by realizing different patterns. However, also the reverse may be true: given a set of continuous films with markedly different magnetic properties, they can be made magnetically similar by drawing the same pattern.

This article focuses just on this second aspect, that has been almost never addressed before, although it also represents a way of controlling the magnetic anisotropy, actually.

We studied a set of three Co:Au continuous films grown by sputtering co-deposition - having similar thickness $(\sim 80 \mathrm{~nm})$ and different concentration ratio, so as to include also a pure Co film and a corresponding set of antidot arrays, produced by nanosphere lithography using the very same scheme.

It is worth remarking that the study of alloys and intermetallic compounds of Au with the magnetic $3 \mathrm{~d}$ elements is of great scientific interest both for the synthesis aspects and for elucidating the physical properties (magnetic, magneto-optical, magneto-plasmonic, catalytic) and envisaging prospective technological applications. ${ }^{31,32,33,34,35}$

Indeed, the aim of our research work - pursued by combining SQUID magnetometry results and atomic and magnetic force microscopy observations - was twofold and, accordingly, the article is substantially divided in two parts. In the first part, the continuous films are addressed, which exhibited a variety of hysteretic properties and magnetic domain configurations. Our study allowed us to assess the magnetization of the films, the effective macroscopic anisotropy and to gain information on the magnetostriction and exchange stiffness of the CoAu compound, thus expanding the knowledge on this particular system. Although it was virtually impossible to disentangle and to estimate the competing anisotropy contributions in the films, the magnetic results highlighted the crucial role of a magnetoelastic term in determining the overall magnetic behavior. The second part of the article deals with the morphological and magnetic properties of the antidot arrays. We will demonstrate, also with the important support of micromagnetic simulations, that the drawn pattern, by raising the shape anisotropy term over the others, induced a similar spin arrangement in the three samples, which resulted in a similar hysteretic behavior. 


\section{Experimental}

Three continuous films were deposited at room temperature on natively oxidized (100)-silicon substrates by magnetron sputtering technique: i) a film with Co:Au concentration ratio 1:1, labelled as $\mathrm{Co}_{1} \mathrm{Au}_{1}$; ii) a film with $\mathrm{Co}: \mathrm{Au}$ concentration ratio 2:1, labelled as $\mathrm{Co}_{2} \mathrm{Au}_{1}$ and iii) a Co film. In the used custom built sputtering apparatus, the $\mathrm{Co}$ and Au targets were tilted of $\sim 30^{\circ}$ with respect to the axis normal to the sample plane, allowing a simultaneous deposition of the two metals. A rotating sample-holder was employed, grounded to the deposition chamber, so as to favor a uniform coverage of the substrate. Argon was the process gas (working pressure $=5 \times 10^{-3} \mathrm{mbar}$ ) and the deposition rate was $\sim 1.5 \AA / s$.

The nominal thickness of the three films was $(80 \pm 5) \mathrm{nm}$; the exact composition of the CoAu films was determined by Rutherford Backscattering Spectrometry (Table 1).

The patterned samples were fabricated by nanosphere lithography, a well-established and powerful method for the manufacturing of nanostructures. ${ }^{36,37}$ In particular, antidot arrays were produced by a standardized procedure, described in more detail elsewhere, ${ }^{38,39,40}$ based on four steps: 1) preparation of a colloidal mask by self-organization of polystyrene nanospheres in a $2 \mathrm{D}$ hexagonalclose-packed lattice; 2) reactive ion etching, leading to a reduction of the diameter of the

Table 1. The data are relative to the continuous films labelled as indicated in Column 1. Columns 2 and 3: Co and Au content. Column 4: saturation magnetization $\mathrm{M}_{\mathrm{S}}$ measured at $\mathrm{T}=300 \mathrm{~K}$. Column 4: shape anisotropy coefficient. Columns 5 and 6. Effective in-plane (IP) and out-of-plane (OP) anisotropies estimated through the analysis of the hysteresis loops in Fig. 1. Column 7: Q parameter.

\begin{tabular}{|c|c|c|c|c|c|c|c|}
\hline \multirow[t]{2}{*}{ Films } & \multicolumn{2}{|c|}{ Composition } & \multirow{2}{*}{$\begin{array}{c}M_{s} \\
\left(e m u / \mathrm{cm}^{3}\right)\end{array}$} & \multirow{2}{*}{$\begin{array}{c}K_{d} \\
\left(\mathrm{erg} / \mathrm{cm}^{3}\right)\end{array}$} & \multirow{2}{*}{$\begin{array}{c}\mathrm{K}_{\mathrm{IP}} \\
\left(\mathrm{erg} / \mathrm{cm}^{3}\right)\end{array}$} & \multirow{2}{*}{$\begin{array}{c}\mathrm{K}_{\mathrm{OP}} \\
\left(\mathrm{erg} / \mathrm{cm}^{3}\right)\end{array}$} & \multirow{2}{*}{$\begin{array}{c}\mathrm{Q}= \\
\mathrm{K}_{\mathrm{OP}} / \mathrm{K}_{\mathrm{d}}\end{array}$} \\
\hline & $\begin{array}{c}\text { Co } \\
\text { (at.\%) }\end{array}$ & $\begin{array}{c}\mathrm{Au} \\
\text { (at.\%) }\end{array}$ & & & & & \\
\hline $\mathrm{Co}_{1} \mathrm{Au}_{1}$ & $53 \pm 1$ & $47 \pm 1$ & $520 \pm 30$ & $\begin{array}{c}(2.0 \pm 0.2) \times \\
10^{6}\end{array}$ & $\begin{array}{c}(2.0 \pm 0.1) \times \\
10^{6}\end{array}$ & - & - \\
\hline $\mathrm{Co}_{2} \mathrm{Au}_{1}$ & $68 \pm 1$ & $32 \pm 1$ & $740 \pm 40$ & $\begin{array}{c}(3.4 \pm 0.4) \times \\
10^{6}\end{array}$ & $\begin{array}{c}(3.5 \pm 0.2) \times \\
10^{6}\end{array}$ & $\begin{array}{c}(1.11 \pm 0.05) \times \\
10^{5}\end{array}$ & $\sim 0.03$ \\
\hline Co & 0 & 100 & $1230 \pm 50$ & $\begin{array}{c}(9.5 \pm 0.8) \times \\
10^{6}\end{array}$ & $\begin{array}{c}(8.6 \pm 0.6) \times \\
10^{6}\end{array}$ & $(2.6 \pm 0.4) \times 10^{6}$ & $\sim 0.3$ \\
\hline
\end{tabular}


nanospheres and, thus, opening the gaps between them; 3) sputtering deposition of the desired material through the mask; 4) removal of the mask, so that the remaining deposited material reflects the symmetry of the open spaces between the nanospheres of the mask.

The nominal initial diameter of the commercial nanospheres (micro-Particles GmbH, Germany), which determined the period of the pattern, was $(522 \pm 12) \mathrm{nm}$. The etching treatment was carried out under an $\mathrm{Ar}-\mathrm{O}_{2}$ atmosphere $\left(30 \% \mathrm{Ar}\right.$ and $\left.70 \% \mathrm{O}_{2}\right)$ at a total pressure of $\sim 370 \times 10^{-3}$ mbar for 7 minutes. After this process, the average diameter of the nanospheres was (280 \pm 9$) \mathrm{nm}$, as estimated by scanning electron microscopy (SEM) observations. ${ }^{40}$

For each composition (Table 1), the 80-nm thick continuous film and the antidot array were grown during the same deposition process, on silicon substrates belonging to the same batch and fixed on the same rotating sample holder.

Atomic force (AFM) and magnetic force (MFM) characterizations were performed with a Bruker Multimode V Nanoscope 8 microscope, equipped with CoCr coated MESP-HR tips, in intermittent contact mode, at a lift scan height of $35 \mathrm{~nm}$. For all images, samples were brought to magnetic remanence after in-plane saturation.

For the magnetic measurements, all the samples were cleaved according to the crystallographic orientation of the substrate, so as to obtain pieces of about $(5 \times 5) \mathrm{mm}^{2}$.

Hysteresis loops were measured on the continuous films at $\mathrm{T}=300 \mathrm{~K}$ using a superconducting quantum interference device (SQUID) magnetometer (maximum applied field $\mathrm{H}=50 \mathrm{kOe}$ ). The loops were corrected for the diamagnetic contributions of the Si substrate.

Micromagnetic simulations were carried out using MuMax3, a GPU-accelerated micromagnetic simulation program developed at Ghent University. ${ }^{41}$

\section{Results and discussion}

\subsection{Magnetic properties of the continuous films}

The values of $\mathrm{M}_{\mathrm{S}}$ measured at $\mathrm{T}=300 \mathrm{~K}$ on the continuous films are reported in Table 1 . Within the errors, they are consistent with those we measured in films with thickness of $\sim 30 \mathrm{~nm}$ and same compositions, sputtered using the same deposition parameters, as we reported in a previous article. ${ }^{33}$ Therefore, we concluded that the structural and compositional properties of the 80-nm thick CoAu films did not differ substantially from those of the thinner films. The latter ones exhibited an amorphous-like structure and consisted of a prevalent CoAu alloyed phase and of segregated cobalt in form of structurally disordered ultrafine particles $(\sim 2 \mathrm{~nm}$ in size $)$ dispersed within the CoAu 
matrix; it was also found out that the percentage of Co in the alloy and the fraction of segregated Co increased with increasing the Co:Au ratio. ${ }^{33,34}$
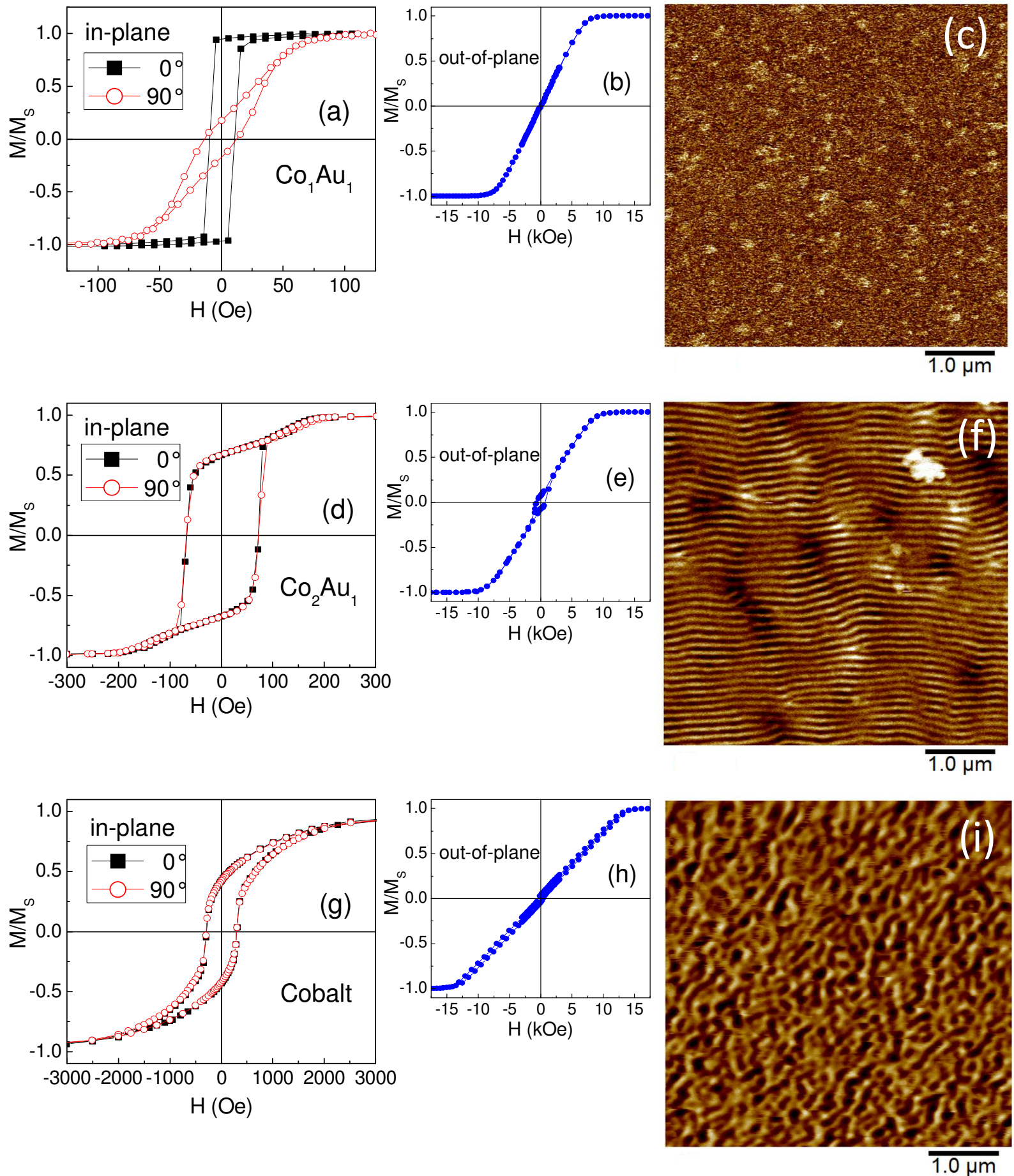

Figure 1. For each of the three investigated continuous films, the in-plane (IP) and out-ofplane (OP) hysteresis loops (normalized to the saturation magnetization $\mathrm{M}_{\mathrm{S}}$ ) and the MFM image (taken at the in-plane magnetic remanence) are displayed along a single row. Frames (a), (b) and (c) refer to $\mathrm{Co}_{1} \mathrm{Au}_{1}$, frames (d), (e) and (f) refer to $\mathrm{Co}_{2} \mathrm{Au}_{1}$ and frames (g), (h) and (i) to pure Co. 
The Co film was nanocrystalline with a dominant hcp structure, ${ }^{42}$ as it is quite usual for sputtered pure Co layers. ${ }^{43}$

Magnetic hysteresis loops were measured on the films at $\mathrm{T}=300 \mathrm{~K}$ : the magnetic field $\mathrm{H}$ was applied along two in-plane (IP) orthogonal directions corresponding to the sides of the sample (conventionally indicated as the $0^{\circ}$ and $90^{\circ}$ directions) and along the normal to the sample plane (out-of-plane, OP); in all the three cases, the magnetization along the direction of $\mathrm{H}$ was recorded. The loops are displayed in Fig. 1 as normalized to the saturation magnetization $\mathrm{M}_{\mathrm{S}}$. Moreover, for each film, typical MFM images, taken at the in-plane magnetic remanence, are also shown in Fig 1.

The sample $\mathrm{Co}_{1} \mathrm{Au}_{1}$ is clearly anisotropic in the plane (Fig. 1a) and the direction $0^{\circ}$, corresponding to the high-remanence loop, is a preferential magnetization axis. The OP loop features a linear anhysteretic trend up to saturation, which indicates that the magnetization vector lies in the film plane and no perpendicular magnetization component exists (Fig. 1b). This is also confirmed by the MFM analysis in Fig. 1c showing an uniform contrast.

The loops measured on $\mathrm{Co}_{2} \mathrm{Au}_{1}$ are shown in Fig. 1d-e. The film is isotropic in the plane. The shape of the IP loops is characteristic of the so-called transcritical hysteretic behavior. This refers to a particular magnetization process involving IP and OP components of the magnetization, typically observed in films with a stripe domains magnetic structure. ${ }^{44,45,46}$ As a matter of fact, the OP loop shows hysteresis at low $\mathrm{H}$ values, indicating the existence of a OP magnetization component and the MFM image definitely confirms the existence of dense stripe domains (Fig. 1f).

The Co film is isotropic in the plane and magnetic hysteresis is observed both in-plane and out-ofplane, indicating that an OP component of the magnetization does exist (Fig. 1g-h). The magnetic contrast of the corresponding MFM image, in Fig. 1i, is indicative of a very complex magnetic configuration, certainly consistent with the existence of a perpendicular anisotropy contribution.

Hence, the three films show completely different magnetic structures, resulting in different hysteretic behaviors.

For a magnetically saturated thin film, the magnetostatic energy density can be expressed as $\mathrm{E}_{\mathrm{ms}}=$ $1 / 2 \mathrm{~N}_{\mathrm{D}} \mathrm{M}_{\mathrm{S}}{ }^{2} \sin ^{2} \phi$, where $\mathrm{N}_{\mathrm{D}}$ is the demagnetizing factor along the direction orthogonal to the plane of the film and $\phi$ is the angle that the $\mathrm{M}_{\mathrm{S}}$ vector forms with the plane of the film. In a thin film, $\mathrm{N}_{\mathrm{D}}$ assumes the maximum possible value of $4 \pi$ and therefore the magnetization vector is forced to lie in the plane, in order to minimize $\mathrm{E}_{\mathrm{ms}}$; hence, the shape anisotropy is expressed by the coefficient $\mathrm{K}_{\mathrm{d}}=2 \pi \mathrm{M}_{\mathrm{S}}{ }^{2}{ }^{7}$ Based on the value of $\mathrm{M}_{\mathrm{S}}$, we have calculated $\mathrm{K}_{\mathrm{d}}$ for the three samples (Table 1). It is known that cobalt is a magnetostrictive material ${ }^{7}$ and, as we also inferred from our previous study on the $30-\mathrm{nm}$ thick films, ${ }^{33}$ the CoAu alloy is likely to possess the same property, possibly 
depending on the Co and Au relative concentrations (no information can be found in literature on this respect). Moreover, it is well known that sputtered films can be subjected to a residual mechanical stress, due to extrinsic factors (for instance, the mismatch in the thermal expansion coefficients of the film and of the substrate) and to intrinsic factors (growth processes, grain structure, substitutional or interstitial impurities). ${ }^{16}$ Hence, the existence of magnetoelastic anisotropy must be considered, whose coefficient is expressed by the relation $K_{m e}=3 / 2 \lambda_{s} \sigma$, where $\lambda_{\mathrm{s}}$ is the magnetostriction and $\sigma$ is the stress; the magnetoelastic anisotropy energy density is $\mathrm{E}_{\mathrm{me}}=$ $\mathrm{K}_{\mathrm{me}} \sin ^{2} \phi$, $\phi$ being the angle between the stress axis and the magnetization vector. ${ }^{7}$

The in-plane anisotropy $\mathrm{K}_{\mathrm{IP}}$ of the three films can be estimated by considering the OP hysteresis loops in Fig. 1b-e-h. For easiness, they are shown together again in Fig. 2, where the first quadrant of the M/M $\mathrm{M}_{\mathrm{S}}$ vs $\mathrm{H}$ plot is displayed. Based on the Stoner Wohlfarth model, the field at which the OP magnetization curve presents a knee corresponds to the anisotropy field $\mathrm{H}_{\mathrm{K}}=2 \mathrm{~K}_{\mathrm{IP}} / \mathrm{M}_{\mathrm{S}}$. The values of $K_{I P}$ are reported in Table 1 . They are very close to the $K_{d}$ values, namely the effective in-plane anisotropy is the shape anisotropy, as expected. Similarly, for the $\mathrm{Co}_{2} \mathrm{Au}_{1}$ and $\mathrm{Co}$ films, we can estimate the out-of-plane anisotropy $\mathrm{K}_{\mathrm{OP}}$ by considering the IP hysteresis loops, in Fig. 1d-g. In $\mathrm{Co}_{2} \mathrm{Au}_{1}, \mathrm{H}_{\mathrm{K}}=2 \mathrm{~K}_{\mathrm{OP}} / \mathrm{M}_{\mathrm{S}}$ is the field where the IP loop exhibits the marked knee. For the Co film, $\mathrm{H}_{\mathrm{K}}$ is not exactly defined. We can assume that it roughly corresponds to the field where the two branches of the hysteresis loop join together. The calculated values of $\mathrm{K}_{\mathrm{OP}}$ are reported in Table 1 together with those of $\mathrm{Q}=\mathrm{K}_{\mathrm{OP}} / \mathrm{K}_{\mathrm{d}}$. This last parameter plays an important role in the description of the magnetic configuration of thin films. ${ }^{47}$

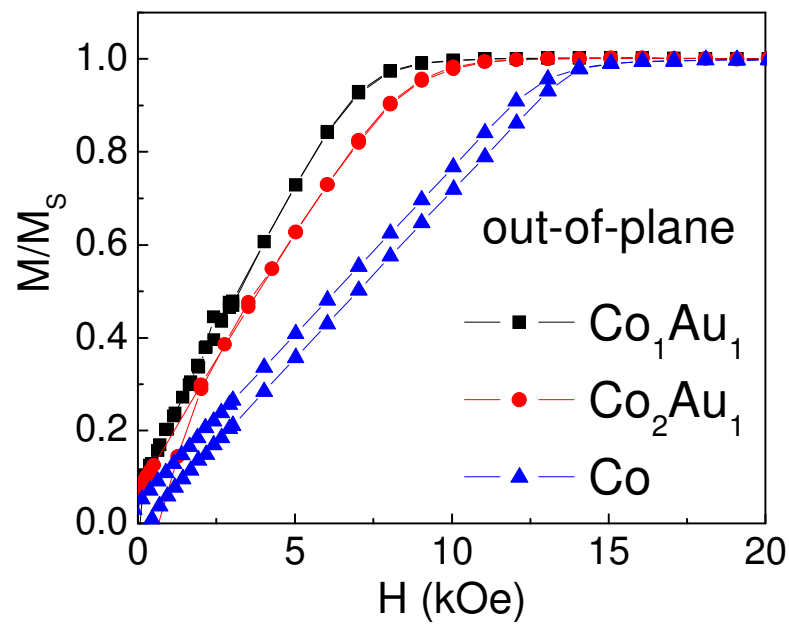

Figure 2. Out-of-plane hysteresis loops for the three continuous films, already shown in Fig. 1. The first quadrant of the $\mathrm{M} / \mathrm{M}_{\mathrm{S}}$ vs. H plot is displayed. 
In the $\mathrm{Co}_{1} \mathrm{Au}_{1}$ film, the magnetization vector lies in the film plane because of shape anisotropy, whereas the existence of a preferential in-plane magnetization direction can be ascribed to the magnetoelastic anisotropy (the magnetocrystalline anisotropy can be disregarded in both the CoAu films, as they are substantially amorphous ${ }^{33,34}$ ). This last effect implies that a non-uniform stress distribution is settled in our films, so that the average stress along two orthogonal directions may be different. We achieved a similar conclusion also in our previous study relative to similar CoAu films with smaller thickness. ${ }^{33}$ Despite several models on the mechanical properties of filmsubstrate systems assume a spatial stress uniformity, ${ }^{48}$ a non-uniform stress distribution is much more likely to occur in practice ${ }^{49}$ due, for instance, to non-ideal isotropic structural properties of the substrate.

In the $\mathrm{Co}_{2} \mathrm{Au}_{1}$ film, a stripe domain pattern is observed. In principle, a thin film with a weak perpendicular anisotropy and $\mathrm{Q}<1$, would be spontaneously magnetized in plane. However, in order to reduce the total anisotropy energy, beyond a critical thickness the magnetization can periodically oscillate out of the plane, which gives rise to the stripe domain configuration. The first observation of this effect was reported by Saito et al., who studied NiFe films with in-plane shape anisotropy and additional perpendicular stress anisotropy. ${ }^{50}$ Since then, the same magnetic domain structure has been observed and studied in a number of different materials, ${ }^{51,52,53,54}$ but very seldom in CoAu samples and, however, only for very low $\mathrm{Au}$ concentration. ${ }^{55} \mathrm{We}$ ascribe the existence of stripe domains in our $\mathrm{Co}_{2} \mathrm{Au}_{1}$ film to the competition between the shape anisotropy and a perpendicular anisotropy term, which necessarily must be magnetoelastic in nature. Hence, in the $\mathrm{Co}_{1} \mathrm{Au}_{1}$ film the magnetoelastic term is effective in the plane whereas in $\mathrm{Co}_{2} \mathrm{Au}_{1}$ it acts orthogonally to the plane.

In the range of $\mathrm{Q}<0.1$, which is the case of the $\mathrm{Co}_{2} \mathrm{Au}_{1}$ film (Table 1), the critical thickness for stripe domains can be approximately calculated by the relation $\mathrm{D}_{\mathrm{cr}}=2 \pi\left(\mathrm{A} / \mathrm{K}_{\mathrm{OP}}\right)^{1 / 2}$, where $\mathrm{A}$ is the magnetic exchange stiffness. ${ }^{47}$ The last parameter is unknown for the investigated compound, but it can be roughly estimated by assuming that $\mathrm{D}_{\text {cr }}$ corresponds to the thickness of the $\mathrm{Co}_{2} \mathrm{Au}_{1}$ film. Under this assumption, a maximum value of $\mathrm{A}$ is calculated, actually. The result is $\mathrm{A} \sim 0.2$ $\mu \mathrm{erg} / \mathrm{cm}$, namely the exchange stiffness in the $\mathrm{Co}_{2} \mathrm{Au}_{1}$ film is one order of magnitude lower compared to bulk cobalt $(\mathrm{A}=3 \mu \mathrm{erg} / \mathrm{cm})$.

The stripe width is expected to be of the same order of the film thickness when $\mathrm{Q}<0.1{ }^{47}$ In quite good agreement with this general rule, the domain width estimated by the MFM analysis on the $\mathrm{Co}_{2} \mathrm{Au}_{1}$ film is $(65 \pm 5) \mathrm{nm}$. 
In the Co film, $\mathrm{Q}$ is smaller than one, but it is definitely larger than in $\mathrm{Co}_{2} \mathrm{Au}_{1}$ and $\mathrm{K}_{\mathrm{OP}}$ increases too (Table 1). If $\mathrm{K}_{\mathrm{OP}}$ were purely magnetoelastic in $\mathrm{Co}$ as in $\mathrm{Co}_{2} \mathrm{Au}_{1}$, one would conclude that the absolute value $|\lambda \sigma|$ increases with the cobalt content, which could suggest a plausible dependence of $\lambda$ on Co. However, it is to be noted that the value of $\mathrm{K}_{\mathrm{OP}}$ in the Co film is of the same order of magnitude of the magnetocrystalline anisotropy coefficient of hcp cobalt $\left(\mathrm{K}_{\mathrm{mc}}=5 \times 10^{6} \mathrm{erg} / \mathrm{cm}^{3}\right)$ and one order larger than in $\mathrm{Co}_{2} \mathrm{Au}_{1}$ (Table 1). This seems to indicate that a net perpendicular magnetocrystalline anisotropy also exists in the nanocrystalline Co film. Hence, the magnetoelastic and magnetocrystalline terms acting in the Co film cannot be disentangled, actually.

We propose that the competition between the magnetoelastic and magnetocrystalline perpendicular anisotropy contributions, counteracted by the in-plane shape term, results in a wide distribution, both in direction and strength, of local anisotropies. This gives rise to the observed complex labyrinthine-type magnetic domain configuration (Fig. 1i). On the contrary, in $\mathrm{Co}_{2} \mathrm{Au}_{1}$, a more uniform perpendicular anisotropy results in a canonical stripe domain arrangement. However, in this film too, the peculiar darker features visible in Fig. 1f - which are not associated to any morphological characteristics of the film, as we verified by AFM - can be tentatively connected to spatial regions where $\sigma$ is markedly different compared to the average value, thus locally modifying the regular stripe configuration. ${ }^{56}$ Indeed, this interesting effect deserves further investigation.

\subsection{Morphology and magnetic properties of the antidot arrays}

Typical AFM images relative to the patterned $\mathrm{Co}_{2} \mathrm{Au}_{1}$ sample are shown in Fig. 3. The AFM analysis on the other two arrays of antidots revealed very similar morphologies. The antidots appear as circular black holes. Their arrangement presents topological defects, as visible in Fig. 3a. One can easily distinguish spatial regions (macroareas), separated by bright and relatively wide boundaries, with a different orientation of the hexagonal lattice. The effect is not unusual for samples prepared by nanosphere lithography since it depends on the degree of ordering of the nanospheres forming the mask. ${ }^{37}$

The thickness of the magnetic film grown at the boundary of the macroareas was estimated by profiling along the green line in Fig. 3a, passing through the boundary and the bottom of a hole adjacent to it: the result is $(65 \pm 5) \mathrm{nm}$. This value is $\sim 19 \%$ smaller than the nominal thickness of the continuous $\mathrm{Co}_{2} \mathrm{Au}_{1}$ film. The same occurs for $\mathrm{Co}_{1} \mathrm{Au}_{1}$ and $\mathrm{Co}$ : the thickness of the film at the boundaries of the macroareas, in the patterned sample, differs from that of the continuous film by 

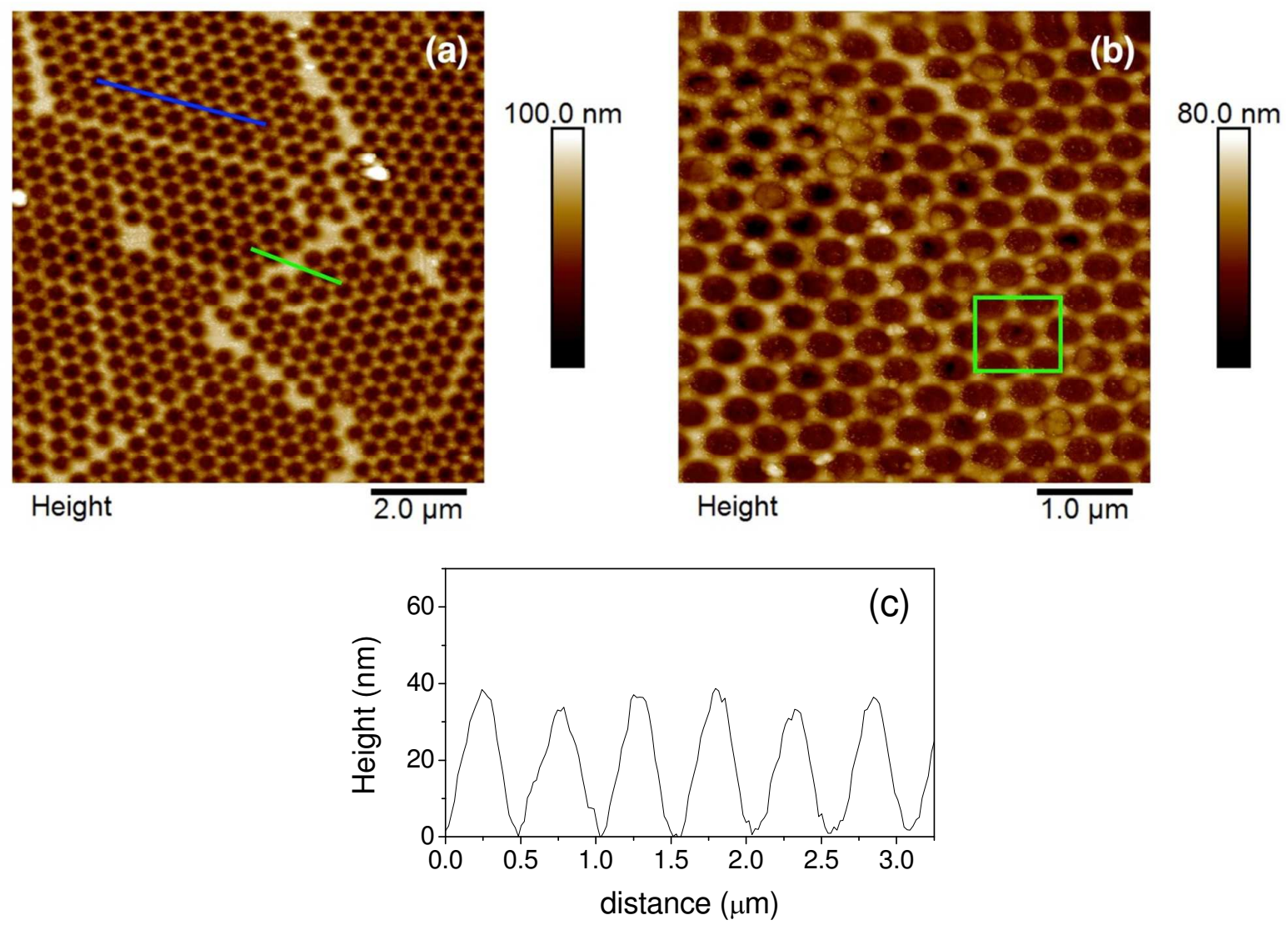

Figure 3. (a) AFM image of the $\mathrm{Co}_{2} \mathrm{Au}_{1}$ antidot array. The green and blue lines represent the profiles along which the thickness of the material has been measured. (b) Another portion of the same sample imaged at a higher magnification. The green square identifies a single hole with the six bright spots surrounding it. (c) Profile of the array along the blue line shown in (a), joining the saddle points.

$15-20 \%$. This reduction is ascribable to shadowing effects induced by the nanospheres during sputtering deposition and by the adopted oblique-incidence deposition geometry. ${ }^{57,58,59}$

The image in Fig. 3b, representing another portion of the same sample at a higher magnification, allows the details of the pattern to be better appreciated. In particular, around each black hole one can distinguish six bright spots (see, for instance, the region framed by the green square), interconnected by thin lines showing a weaker contrast. These features are consistent with the presence, around each hole, of six cusps connected by saddles, so that the final geometry is reminiscent of an egg box (except for the fact that, usually, egg boxes have a square lattice, instead of hexagonal).

Profiling along lines joining the saddle points - an example of this is shown in Fig. 3c for the blue line drawn in Fig. 3a - reveals that the periodicity of the pattern is $\sim 500 \mathrm{~nm}$ (i.e. the initial diameter of the nanospheres), as expected, and the height of the saddles with respect to the bottom of the holes is $\sim 35 \mathrm{~nm}$. Moreover, it is found out that the diameter of the holes decreases moving from 
the top to the bottom of the patterned film since the edge of the deposit is not sharp (i.e., the holes are not cylindrical and the bottom of the holes is flat just in its innermost part). Again, the reason for this is the shadowing effect of the nanospheres during the oblique-incidence deposition in combination with the use of the rotating sample holder. At their maximum height, the cusps are, on the average, slightly thinner than the film grown at the boundaries of the macroareas. In fact, in general, the shadowing effect tends to be more pronounced where the nanospheres are better arranged and more closely packed, i.e. within the macroareas.
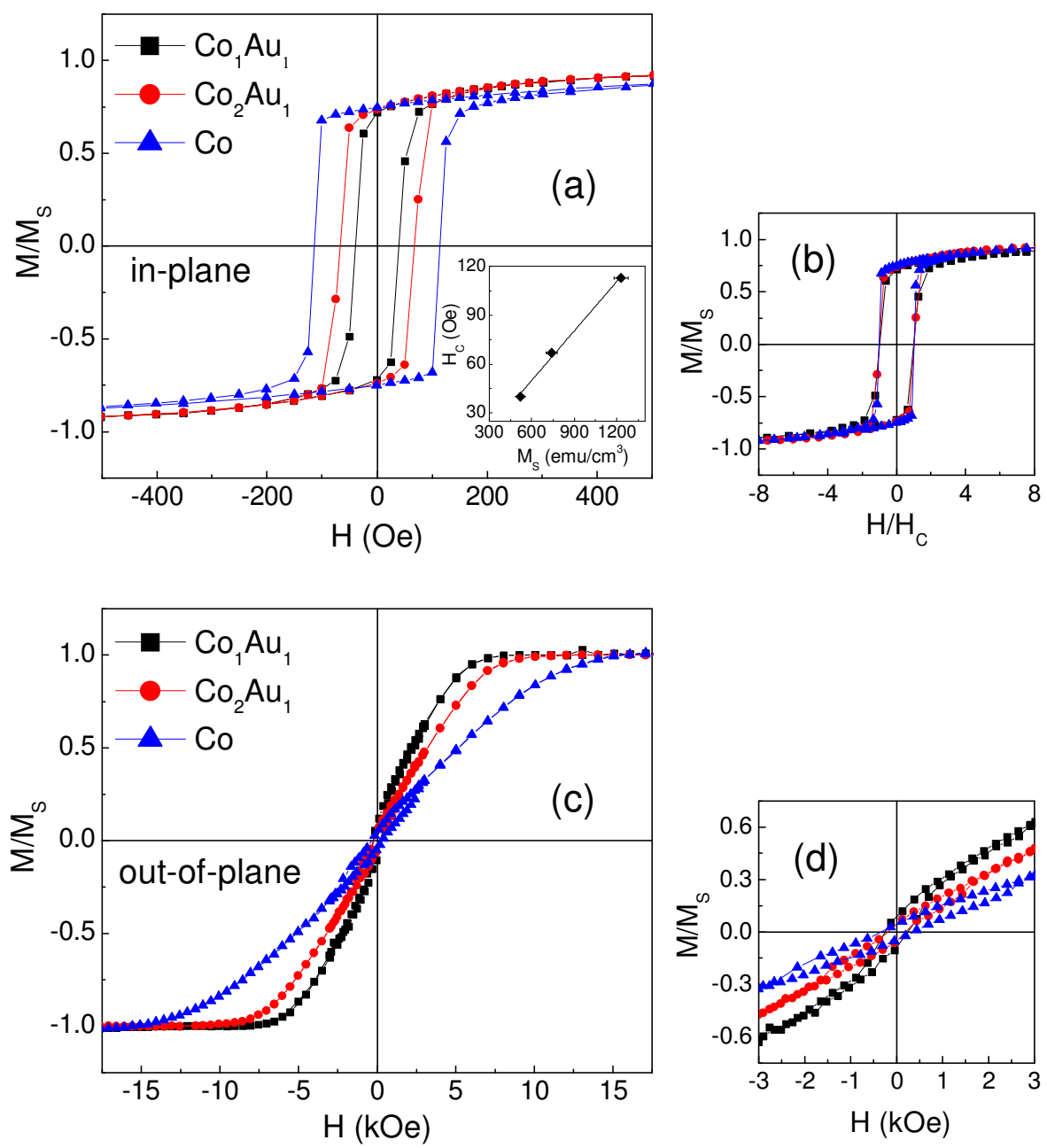

Figure 4. (a) In-plane hysteresis loops measured on the three antidot arrays (normalized to the saturation magnetization $\mathrm{M}_{\mathrm{S}}$ ); in the inset, the coercivity $\mathrm{H}_{\mathrm{C}}$ of the three samples is plotted as a function of $M_{S}$. (b) The in-plane hysteresis loops are shown as $M / M_{S} v s . H / H_{C}$. (c) Out-of-plane hysteresis loops for the three antidot arrays (normalized to $\mathrm{M}_{\mathrm{S}}$ ). (d) is an enlarged view of the loops in (c). 
The IP and OP loops measured at $\mathrm{T}=300 \mathrm{~K}$ on the antidot arrays are shown in Fig. 4. Moreover, IP loops were measured by magneto-optic Kerr effect (MOKE) magnetometry, by applying the magnetic field at different angles $\theta\left(0^{\circ}, 45^{\circ}, 60^{\circ}, 90^{\circ}\right)$ with respect to a conventional $0^{\circ}$ direction, coincident with one edge of the squared samples. It turned out that the patterned films are isotropic in the plane (not shown).

The IP coercivity increases with the Co content and it depends linearly on the value of $\mathrm{M}_{\mathrm{S}}$ reported in Table 1 (inset of Fig. 4a), as expected when the magnetization process is ruled by shape anisotropy. ${ }^{7}$ In general, the shape of the IP loops is very similar for the three patterned films, unlike what observed in the continuous ones. This effect is especially well appreciable if one re-plots the IP loops as a function of $\mathrm{H} / \mathrm{H}_{\mathrm{C}}$, as in Fig. 4b. As for the OP loops, they do exhibit magnetic hysteresis, revealing the existence of an out-of-plane magnetization component in the three cases (Fig. 4c-d). This last statement is confirmed by the MFM analysis shown in Fig. 5: the images (taken at the in-plane magnetic remanence) are characterized by a marked magnetic contrast and, in general, reveal complex magnetic configurations.

In order to correlate the magnetic configuration to the morphological structure, the MFM and AFM images relative to the very same portion of the Co array are compared in Fig 5a-b. On the AFM image, we have drawn small white and blue circles to highlight some cusps and green circles to highlight some holes. Then, the very same sketches have been moved on the corresponding positions on the MFM image. It turns out that the darkest regions in the MFM map are produced by the cusps, whereas the bright contrast is observed at the holes.

An accurate inspection of the MFM images of the $\mathrm{Co}_{1} \mathrm{Au}_{1}$ and $\mathrm{Co}_{2} \mathrm{Au}_{1}$ arrays (Fig. 5c-d) allows one to recognize some similarities with the magnetic configuration of the Co sample. For instance, we focus on the geometrical scheme (for easiness, drawn in Fig. 5e) formed by the seven green circles that in Fig. 5b point out the holes: the same scheme can be distinguished also in Fig. 5c-d (to better highlight it, it has been enclosed in a circular frame), though with increasing difficulty as the Co content in the samples decreases.

Therefore, the magnetic structure of the three antidot arrays is somewhat similar, consistently with their similar hysteretic behavior. Hence, in these samples, the magnetoelastic anisotropy does not play the same crucial role as in the continuous films, where it mainly contributes to setting very different magnetic configurations and hysteretic properties. In this respect, one should also consider that the nanopatterning may induce a substantial reduction of the residual stresses in the antidot arrays and/or of the spatial length on which they act. 


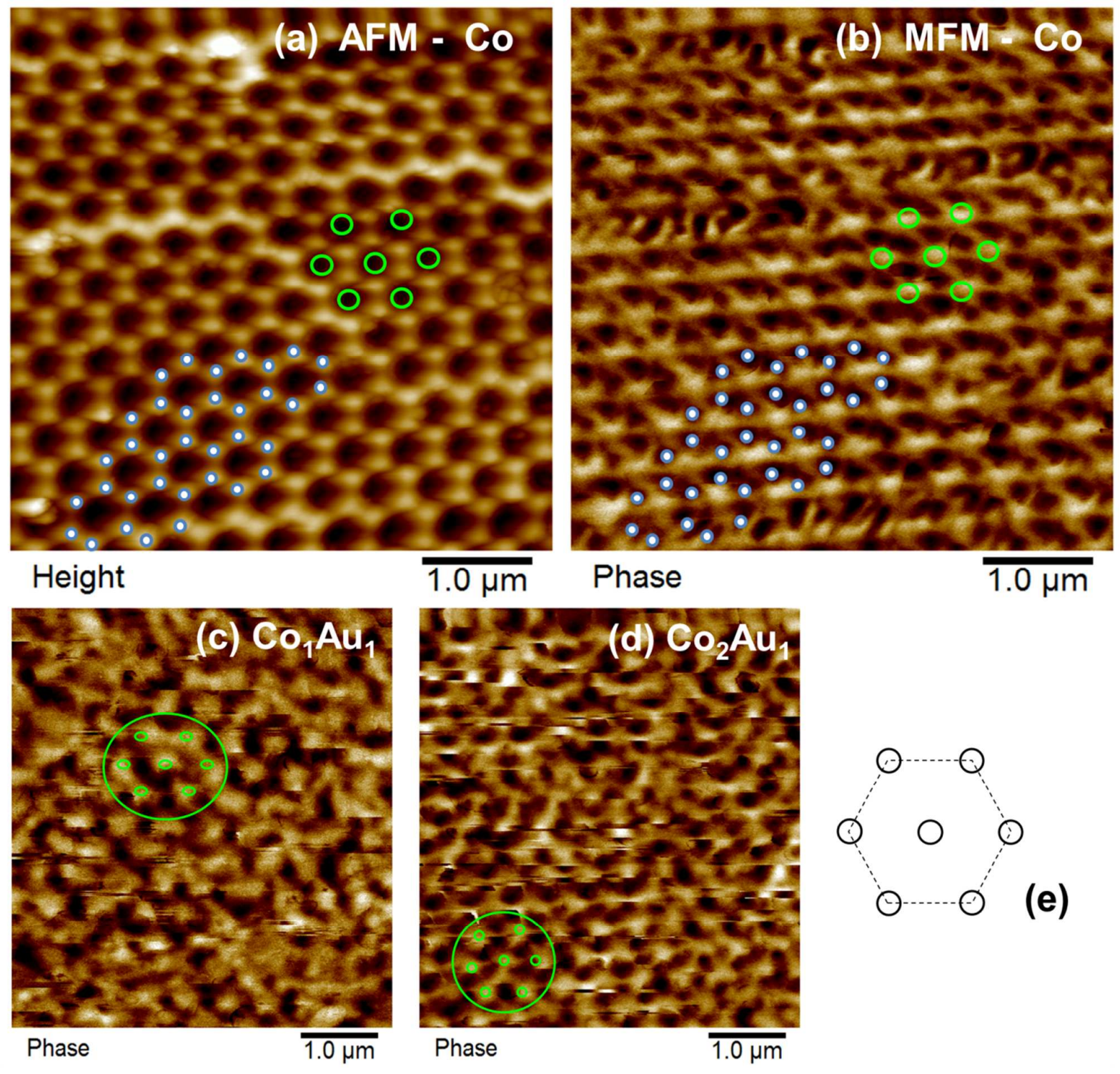

Figure 5. AFM (a) and MFM (b) images for the Co antidot array: the white and blue points indicate the positions of the cusps and the green circles highlight the holes. The scheme formed by these green circles, sketched in frame (e), is also visible in the MFM images of the $\mathrm{Co}_{1} \mathrm{Au}_{1}$ (c) and $\mathrm{Co}_{2} \mathrm{Au}_{1}$ (d) arrays (to better highlight it, it has been enclosed in a circular frame). See text for explanation.

The anisotropic term certainly present in the three arrays is the shape one. Obviously, compared to the continuous films, the shape factor, and hence $\mathrm{K}_{\mathrm{d}}$, is locally modulated by the nanopatterning. The IP loops in Fig. $4 \mathrm{a}$ and the observed linear dependence of $\mathrm{H}_{\mathrm{C}}$ on $\mathrm{M}_{\mathrm{S}}$ indicate that the shape anisotropy forces the magnetization vector in the plane of the patterned films. On the other hand, the existence of magnetic hysteresis in the OP loops (Fig. 4d) suggests that the magnetization may be pushed out-of-plane, probably in correspondence of the cusps imaged by AFM. 


\subsection{Micromagnetic simulations of the antidot arrays}

In order to gain more information on the magnetic configuration of the antidot arrays and account for the MFM images and the shape of the hysteresis loops, we carried out micromagnetic simulations by MuMax3, exploiting the finite difference method. This requires a discretization of a chosen geometric figure over a grid of identical prism-cells in which the magnetization is assumed to be uniform. Hence, we designed the geometric figure shown in Fig. 6a. Please note that, in Fig. 6 , we have deliberately expanded the chosen geometry by a factor of 5 along the $\mathrm{z}$ direction for better viewing. In order to avoid finite size effects, the calculations were carried out using 2D periodic boundary conditions, which means that the geometry in Fig. 6a is replicated in the $x-y$ plane so as to simulate an infinite film. To better visualize the pattern obtained in this way, four replicas are displayed in Fig. 6b. The basic geometry was discretized using cells with basis of $(4 \times$ 4) $\mathrm{nm}^{2}$ and height $4 \mathrm{~nm}$. In the $\mathrm{x}-\mathrm{y}$ plane, the figure occupies an area of $(130 \times 225)$ cells, namely $(520 \times 900) \mathrm{nm}^{2}$. Along $\mathrm{z}$, it consists of 16 superposed layers of cells. In each layer, the cells are arranged so as to reproduce the main features of the real pattern, as revealed by the AFM analysis (Fig. 3). The bottom layer of cells is almost continuous, except for the presence of holes with diameter of $40 \mathrm{~nm}$; the number of cells decreases progressively in the subsequent layers, namely moving towards the top.
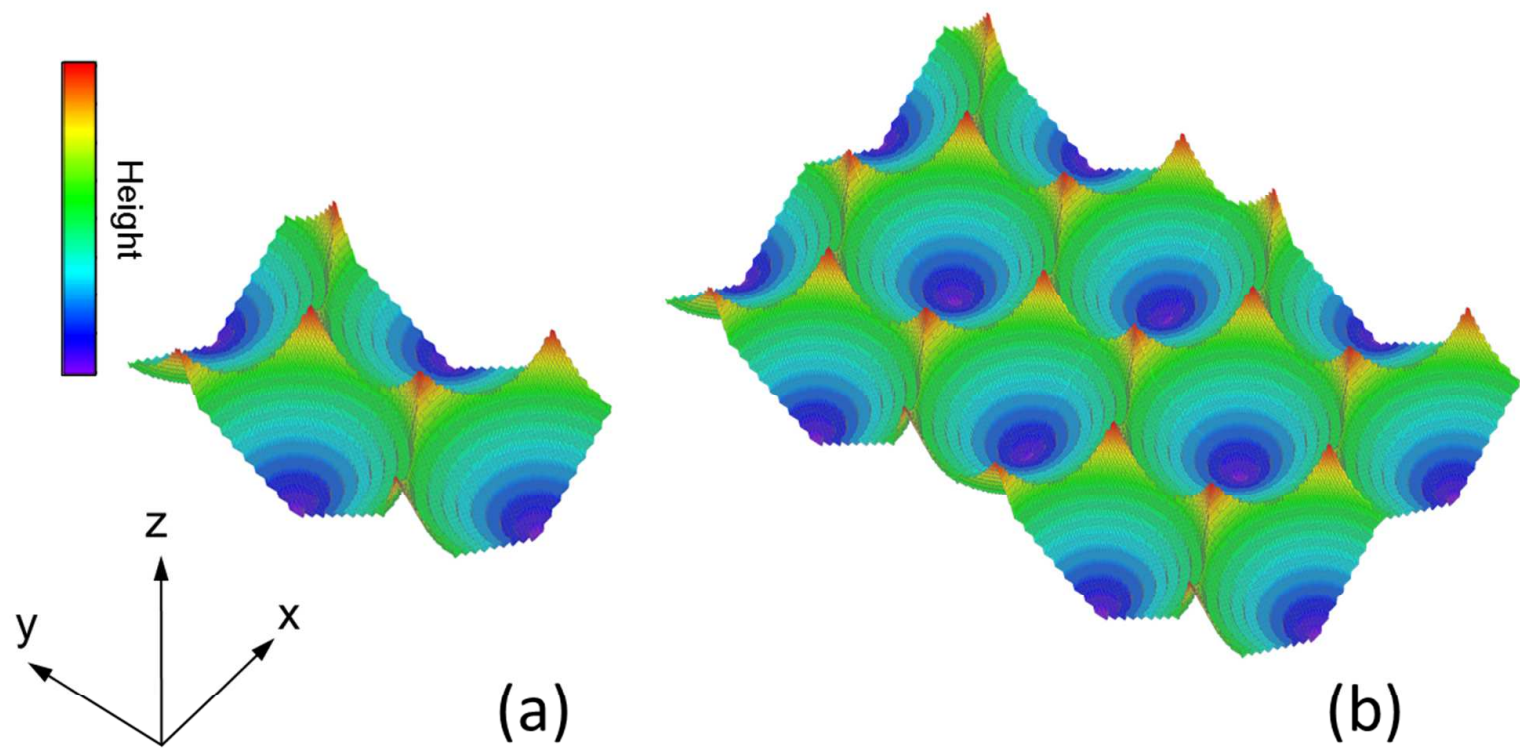

(b)

Figure 6. (a) 3D view of the basic geometric figure simulated in the micromagnetic analysis (expanded by a factor of 5 along the $\mathrm{z}$ direction for better viewing). The scale of colors, from violet to red, indicates the height of the structure along $\mathrm{z}$ (see the text for further explanations). In (b) the basic figure has been replicated four times. 
The top layer contains only the cells that form the final portion of the cusps ( 8 cells for each cusp). The height of the cusps is $64 \mathrm{~nm}$, whereas the height at the saddle point is $36 \mathrm{~nm}$, in line with the AFM results (Fig. 3). Obviously, although we intended to reproduce as closely as possible the array morphology, the geometry in Fig. 6 is an ideal system totally excluding the existence of topological defects and thickness inhomogeneity.

Since we aimed to ascertain, in particular, the dominant role of the shape anisotropy in ruling the magnetic properties of the nanopatterned films, we simulated the case of sample $\mathrm{Co}_{2} \mathrm{Au}_{1}$, in which we excluded the presence of a magnetocrystalline anisotropy term. For the $\mathrm{Co}_{2} \mathrm{Au}_{1}$ composition, we determined the $\mathrm{M}_{\mathrm{S}}$ value (Table 1) and we were able to estimate the order of magnitude of the magnetic exchange stiffness constant A (Section 3.1). Both the parameters are requested for performing micromagnetic calculations.

We carried out simulations of hysteresis loops measured by applying the magnetic field $\mathrm{H}$ in the $\mathrm{x}-\mathrm{y}$ plane at different angles $\theta$ with respect to the $\mathrm{x}$ axis, between $0^{\circ}$ (i.e. $\mathrm{H}$ parallel to the $\mathrm{x}$ axis) and $60^{\circ}$ (the magnetization along the same direction of $\mathrm{H}$ was calculated). The results are shown in Fig. 7a. Both the coercivity and the squareness (i.e., $\mathrm{M}_{\mathrm{r}} / \mathrm{M}_{\mathrm{S}}$, being $\mathrm{M}_{\mathrm{r}}$ the remanent magnetization) decrease with increasing $\theta$ from $0^{\circ}$ to $30^{\circ}$; the loops at $\theta=15^{\circ}$ and $45^{\circ}$ and those at $\theta=0^{\circ}$ and $60^{\circ}$ are perfectly superposed. This is fully consistent with the hexagonal symmetry of the lattice of the antidot array and, in agreement with what reported for antidot arrays with this symmetry, ${ }^{60}$ the easy axes are along the directions where the holes are closest to each other, namely at $\theta=0^{\circ}$ and multiples of $60^{\circ}$.

Hence, the simulated system is anisotropic in the $x-y$ plane, whereas the real sample resulted isotropic in the plane. That is explained considering the presence of the structural macroareas revealed by the AFM analysis (Fig. 3a): due to the different orientation of the hexagonal lattice within each macroarea, the system as a whole does not exhibit hexagonal symmetry. Accordingly, the hysteresis loop measured on the real sample should not be directly compared to any of the simulated loops in Fig. 7a. ${ }^{61}$ A possible approach is to compare the experimental loop (already shown in Fig. 4a) to that obtained by averaging loops calculated for $0^{\circ} \leq \theta<60^{\circ}$. We did that considering 12 loops calculated at values of $\theta$ differing for $5^{\circ}$; we attributed to each loop the same statistical weight, which means modelling the sample as formed by 12 macroareas with the very same extension. The result is shown in Fig. $7 \mathrm{~b}$. The coercivity and the squareness are slightly higher in the simulated loop and the approach to saturation is more rapid. However, in general, the two curves have a pretty similar shape, as one can realize by plotting them as a function of $\mathrm{H} / \mathrm{H}_{\mathrm{C}}$ (inset of Fig. 7b). 

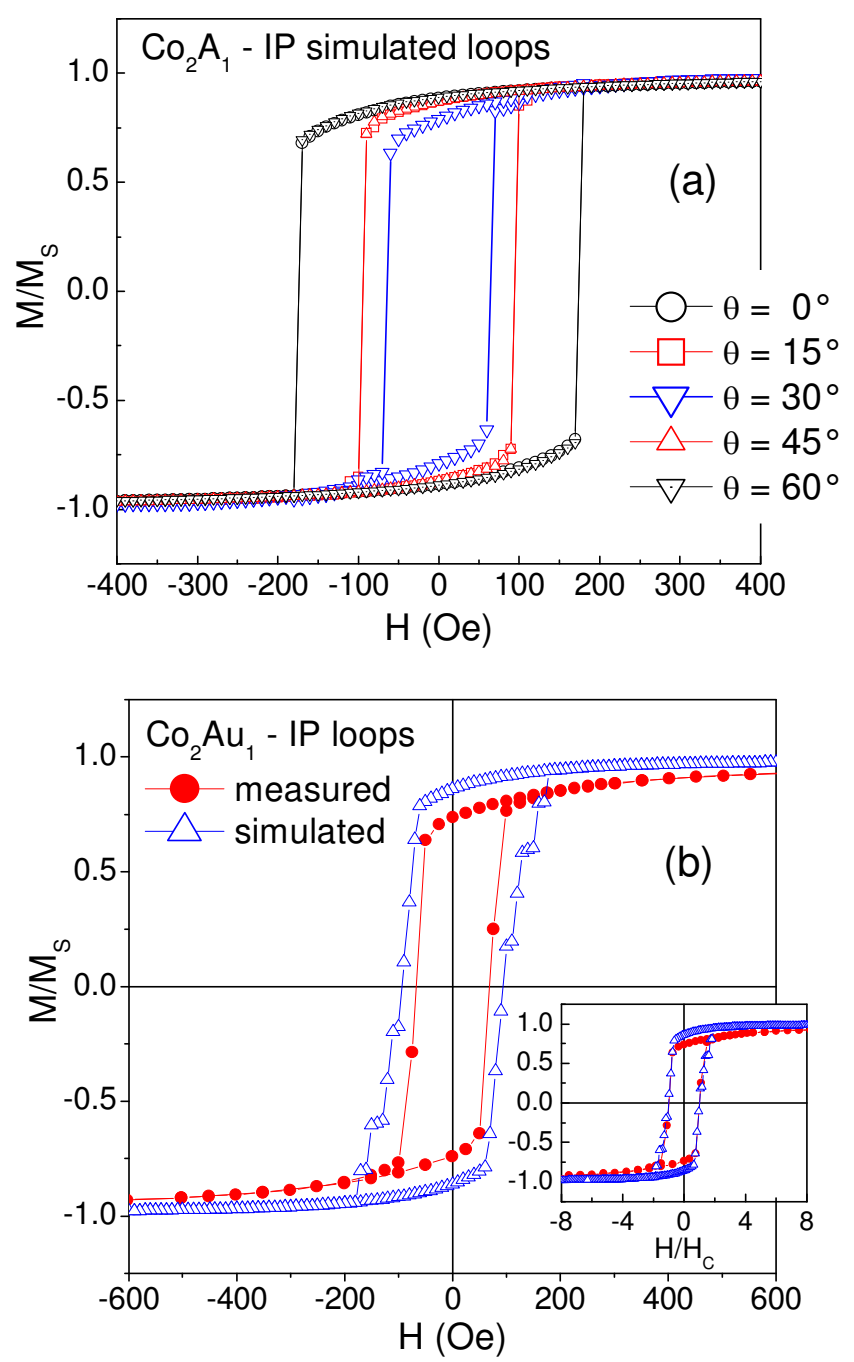

Figure 7. (a) In-plane (IP) simulated loops (normalized to the saturation magnetization $\mathrm{M}_{\mathrm{S}}$ ) for the $\mathrm{Co}_{2} \mathrm{Au}_{1}$ antidot array, calculated at the indicated values of the angle $\theta$ that the applied magnetic field $\mathrm{H}$ forms with the $\mathrm{x}$ axis. The loops for $\theta=0^{\circ}$ and $60^{\circ}$ and for $\theta=$ $15^{\circ}$ and $45^{\circ}$ are perfectly superposed. (b) Measured and simulated IP hysteresis loops for the $\mathrm{Co}_{2} \mathrm{Au}_{1}$ antidot array. The simulated curve is the average of 12 loops calculated for $0^{\circ} \leq$ $\theta<60^{\circ}$. In the inset, the two curves are shown as $\mathrm{M} / \mathrm{M}_{\mathrm{S}} \mathrm{vs} . \mathrm{H} / \mathrm{H}_{\mathrm{C}}$. See the text for further details.

In a simulated hysteresis loop, each point corresponds to a specific configuration of the magnetization (indicated as magnetization map) of the system modeled by the geometric figure in Fig. 6a. In Fig. 8, we show two magnetization maps, corresponding to the positive remanence state (i.e. the state at $\mathrm{H}=0$, after the positive saturation) in the loop at $\theta=0^{\circ}$ of Fig. 7a.

In particular, Fig. 8a is the $\mathrm{x}-\mathrm{y}$ map visible by sectioning the simulated structure along the yellow plane displayed in Fig. $8 \mathrm{~b}$ (the arrows indicate the orientation of the component of the magnetization in the plane); Fig. 8c is the $\mathrm{y}-\mathrm{z}$ map obtained by sectioning along the yellow plane in 
Fig. 8d. In both maps, the cell color provides information about the projection of the magnetization along $\mathrm{z}\left(\mathrm{M}_{\mathrm{z}}\right)$ : blue and red designate the sign of $\mathrm{M}_{\mathrm{z}}$ (positive and negative, respectively) and the color intensity is proportional to $\left|\mathrm{M}_{\mathrm{z}} / \mathrm{M}_{\mathrm{S}}\right|$.

One can see that, especially at the bottom of the array, the magnetization mostly lies in the sample plane, but, since it is forced to rotate around the holes, complex and quite regular magnetic charge distributions appear at the edges. ${ }^{60,62}$ At the cusps, the magnetization tends to align along the $\mathrm{z}$ axis. In fact, with respect to the continuous films, the nanopatterning strongly changed the balance among the demagnetizing factors along the three space directions and, in particular, it caused a significant reduction of the one along the vertical axis of the cusps.
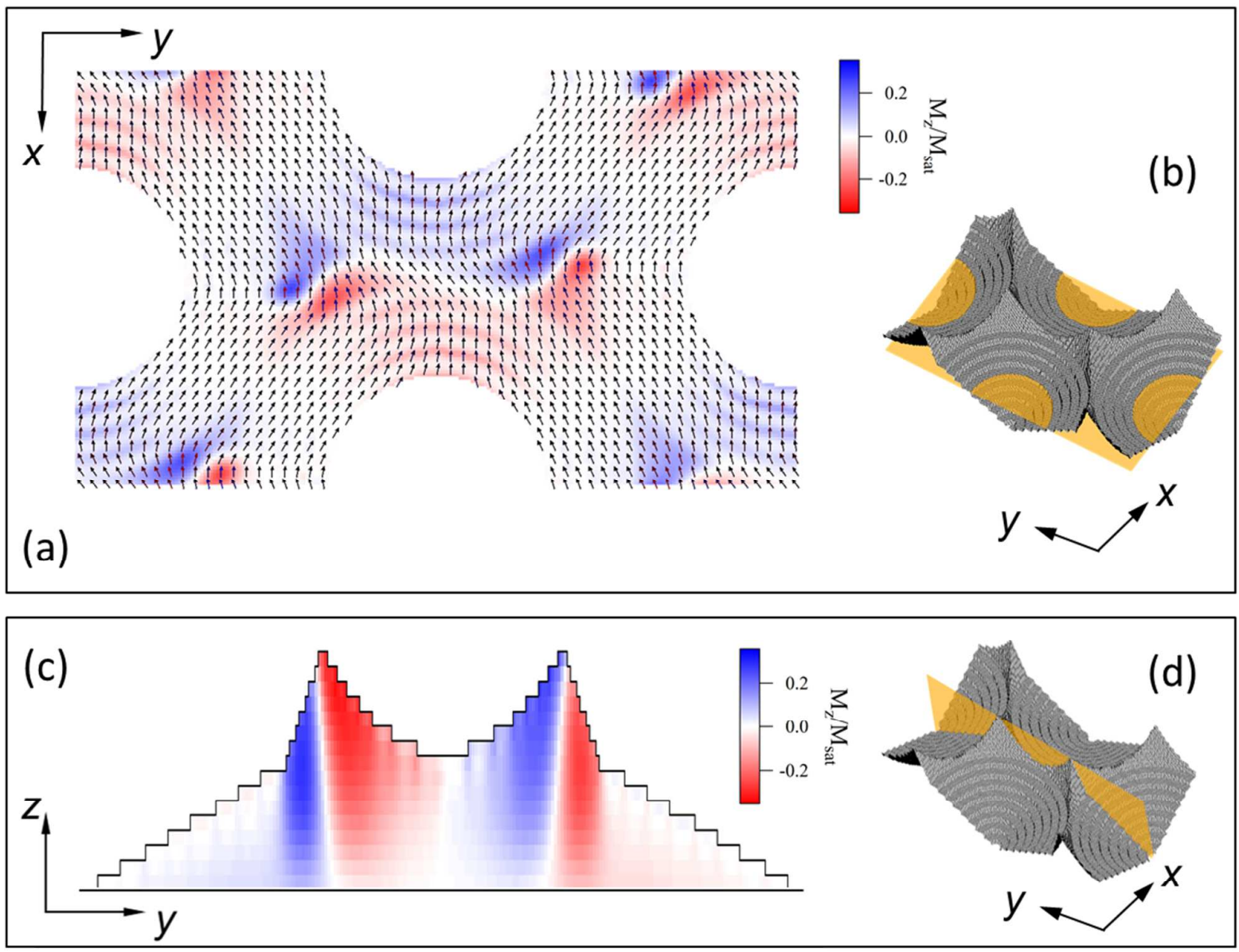

Figure 8. (a) Magnetization $x-y$ map obtained by sectioning the simulated structure along the yellow plane displayed in (b); the arrows indicate the orientation of the component of the magnetization in the plane. (c) Magnetization $y-z$ map obtained by sectioning the simulated structure along the yellow plane displayed in (d); this map has been expanded by a factor of 5 along $\mathrm{z}$ for better viewing. The blue and red colors designate the sign of $\mathrm{M}_{\mathrm{z}}$ (positive and negative, respectively) and the intensity is proportional to $\left|M_{z} / M_{S}\right|$. 
The map in Fig. $8 \mathrm{~b}$ also shows that in each cusp two regions can be distinguished with $\mathrm{M}_{\mathrm{z}}$ pointing upward and downward.

To the MFM (Fig. 5), the cusps appear as dark spots because the vertical component of the magnetic field fringing from the cusps strongly decreases on increasing the distance from the sample surface. ${ }^{63}$ This results in a significant attraction force experienced by the MFM tip, that cannot resolve the individual upward and downward pointing magnetizations since the size of the cusps is of the same order of that of the tip itself, typically 30-40 nm. As a consequence, dark spots are imaged, whereas a relatively brighter contrast is shown in correspondence of the holes, where free poles at the hole edges are responsible for significant fringing fields in the volume above the sample surface.

Then, we carried out micromagnetic simulations also for the $\mathrm{Co}_{1} \mathrm{Au}_{1}$ and $\mathrm{Co}$ arrays. The values of $\mathrm{M}_{\mathrm{S}}$ for these compositions are reported in Table 1, but those of the exchange stiffness A were not exactly known. Therefore, for the Co sample we used the value usually attributed to the bulk hcp phase $(\mathrm{A}=3 \mu \mathrm{erg} / \mathrm{cm})$.
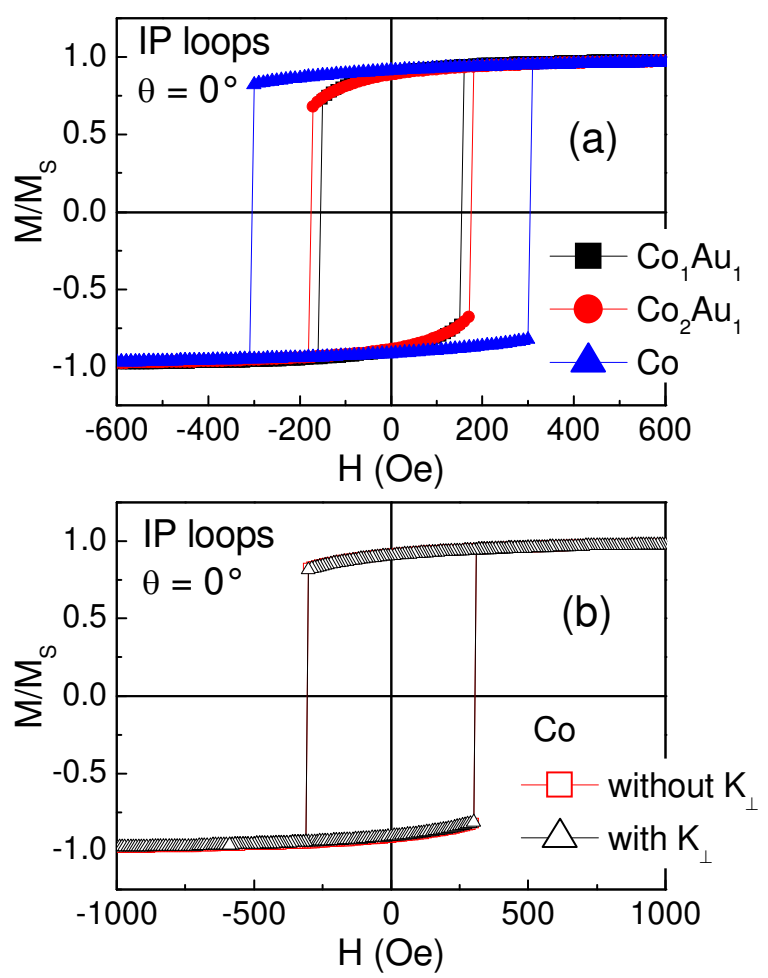

Figure 9. (a) IP simulated loops, calculated at $\theta=0^{\circ}$, for the $\mathrm{Co}_{1} \mathrm{Au}_{1}, \mathrm{Co}_{2} \mathrm{Au}_{1}$ (curve already shown in Fig. 7a) and Co antidot arrays (normalized to the saturation magnetization $\mathrm{M}_{\mathrm{S}}$ ). (b) IP simulated loops $\left(\theta=0^{\circ}\right)$ for the Co array: the curve shown in (a) is compared to that obtained by including an anisotropy term $\mathrm{K}_{\perp}$ in the micromagnetic calculation. The two curves are almost perfectly superposed. 
In the case of $\mathrm{Au}_{1} \mathrm{Co}_{1}$, the choice of $\mathrm{A}$ was much more arbitrary and we tentatively assigned the same value used for $\mathrm{Co}_{2} \mathrm{Au}_{1}$. The magnetization maps calculated at the positive remanence for $\theta=$ $0^{\circ}$ were similar to those for $\mathrm{Co}_{2} \mathrm{Au}_{1}$ in Fig. 8 and the hysteresis loops were similar too. They are displayed, for the three samples, in Fig. 9a. The coercivity decreases with decreasing the Co content in the samples, as observed in the measured loops (Fig. 4a), even if higher $\mathrm{H}_{\mathrm{C}}$ values are calculated for each composition, very likely because they are not obtained by averaging over different values of $\theta$, as we did in the case shown in Fig. 7b.

Regarding the Co array, besides the simulation just described, we carried out another simulation including an anisotropy term directed along $\mathrm{z}$. That was done since for the Co continuous film we hypothesized the existence of a net perpendicular magnetocrystalline anisotropy contribution and we intended to ascertain the possible influence of this term in the observed magnetic behavior of the patterned sample. Therefore, the coefficient assigned to this anisotropy term was $\mathrm{K}_{\perp}=2.6 \times 10^{6}$ $\mathrm{erg} / \mathrm{cm}^{3}$, equal to the value of $\mathrm{K}_{\mathrm{OP}}$ reported in Table 1 . The hysteresis loops calculated at $\theta=0^{\circ}$ with and without $\mathrm{K}_{\perp}$ are shown in Fig. 9b: they are substantially indistinguishable, which confirms once more the dominant role of shape anisotropy.

\section{Conclusions}

We have reported about the magnetic properties of continuous $\mathrm{Co}_{1} \mathrm{Au}_{1}, \mathrm{Co}_{2} \mathrm{Au}_{1}$ and $\mathrm{Co}$ films grown by co-sputtering deposition and of antidot arrays, with same composition, prepared by nanosphere lithography.

The study of the continuous films has allowed us to acquire valuable information on the magnetism of the CoAu compound (magnetization, effective magnetic anisotropy, exchange stiffness constant, magnetostrictive behavior). Moreover, a relationship has been established between the magnetic domain structure of the continuous films and the hysteretic properties, which were found to vary strongly with the composition. Through this approach, we have gained an insight into the interplay between different anisotropy terms, which ultimately rules the magnetic behavior of the films. In particular, we have stressed the role of a magnetoelastic anisotropy term. In $\mathrm{Co}_{1} \mathrm{Au}_{1}$ this term is effective in the plane and, together with shape anisotropy, determines an in-plane anisotropic hysteretic behavior. In $\mathrm{Co}_{2} \mathrm{Au}_{1}$, the competition between the shape and the magnetoelastic terms gives rise to stripe domains and transcritical hysteretic behavior. In Co, the existence of an 
additional perpendicular magnetocrystalline term has been inferred, which may account for the different magnetic structure, labyrinthine-type, compared to $\mathrm{Co}_{2} \mathrm{Au}_{1}$.

In the antidot arrays - having a hexagonal lattice and featuring six cusps around each hole - the anisotropies interplay was strongly modified by the nanopatterning, compared to the continuous films. To elucidate this effect, the experimental findings were synergically coupled to micromagnetic simulations on an ideal model system reproducing as closely as possible the morphology of the arrays. We have found out that the spatially-modulated shape anisotropy is the dominant term and, since the same pattern was used for the three arrays, their spin configurations present a similar scheme, although more difficult to be detected with decreasing the Co content. Accordingly, also the hysteretic behavior of the three patterned films is similar. In particular, in all the three arrays, the shape anisotropy pushes the magnetization out-of-plane in correspondence of the cusps.

Hence, the nanopatterning smoothed out the magnetic differences which characterized the continuous films, thus confirming to be a powerful tool for tailoring the magnetic properties of the magnetic systems at the nanoscale.

\section{Conflicts of interest}

There are no conflicts to declare.

\section{References}

${ }^{1}$ S. Mangin, D. Ravelosona, J.A.Katine, M.J.Carey, B.D.Terris, E. E. Fullerton, Current-induced magnetization reversal in nanopillars with perpendicular anisotropy, Nature Mater., 2006, 5, 210, doi:10.1038/nmat1595

${ }^{2}$ M. Albrecht, G. Hu, I. L. Guhr, T.C. Ulbrich, J. Boneberg, P. Leiderer, G.Schatz, Magnetic multilayers on nanospheres, Nature Mater., 2005, 4, 203, doi:10.1038/nmat1324

${ }^{3}$ B. Dieny, V. S. Speriosu, S. S. P. Parkin, B. A. Gurney, D. R. Wilhoit, D. Mauri, Giant magnetoresistive in soft ferromagnetic multilayers, Phys. Rev. B, 1991, 43, 1297, doi: 10.1103/PhysRevB.43.1297

${ }^{4}$ E.E. Fullerton, J.S. Jiang, S.D. Bader, Hard/soft magnetic heterostructures: model exchange-spring magnets, J. Magn. Magn. Mater., 1999, 200, 392, doi: 10.1016/S0304-8853(99)00376-5

${ }^{5}$ S. Iwasaki, Perpendicular Magnetic Recording Focused on the Origin and Its Significance, IEEE Trans. Magn., 2002, 38, 1609, 10.1109/TMAG.2002.1017743

${ }^{6}$ M. Farle, B. Mirwald-Schulz, A. N. Anisimov, W. Platow, K. Baberschke, Higher-order magnetic anisotropies and the nature of the spin-reorientation transition, Phys. Rev. B, 1997, 55, 3708, doi: 10.1103/PhysRevB.55.3708 
${ }^{7}$ B.D. Cullity, C.D. Graham, Introduction to Magnetism and Magnetic Materials, 2nd ed; IEEE Press, Piscataway NJ, USA Wiley, 2009

${ }^{8}$ R. Alben, J.J. Becker, M.C. Chi, Random anisotropy in amorphous ferromagnets, J. Appl. Phys., 1978, 49, 1653, doi: 10.1063/1.324881

${ }^{9}$ Y. Yoshizawa, S. Oguma, K. Yamauchi, New Fe-based soft magnetic alloys composed of ultrafine grain structure, J. Appl. Phys., 1988, 64, 6044, doi: 10.1063/1.342149

${ }^{10} \mathrm{G}$. Herzer, Grain size dependence of coercivity and permeability in nanocrystalline ferromagnets. IEEE Trans. Magn., 1990, 26, 1397, doi: 10.1109/20.104389.

${ }^{11}$ A Hernando, M Vázquez, J M Barandiaran, Metallic glasses and sensing applications, J. Phys. E: Sci. Instrum., 1988, 21, 1129, doi: 10.1088/0022-3735/21/12/002

${ }^{12}$ E. Bonetti, L. Del Bianco, P. Tiberto, Anelastic and magnetoelastic effects and microstructural evolution of the $\mathrm{Fe}_{73.5} \mathrm{Cu}_{1} \mathrm{Nb}_{3} \mathrm{Si}_{13.5} \mathrm{~B}_{9}$ alloy, J. Magn. Magn. Mater., 1995, 140-144, 477, doi: 10.1016/0304-8853(94)00736-5

${ }^{13}$ G.V. Kurlyandskaya, M. Vázquez, J.L. Muñoz, D. García, J. McCord, Effect of induced magnetic anisotropy and domain structure features on magnetoimpedance in stress annealed Co-rich amorphous ribbons, J. Magn. Magn. Mater., 1999, 196-197, 259, doi: 10.1016/S03048853(98)00805-1

14 C.C. Koch, Synthesis of nanostructured materials by mechanical milling: problems and opportunities, Nanostruct. Mater., 1997, 9, 13, doi: 10.1016/S0965-9773(97)00014-7

${ }^{15}$ L. Del Bianco, F. Spizzo, M. Tamisari, Tailoring the exchange bias of Ni/NiO nanogranular samples by the structure control, J. Magn. Magn. Mater., 2009, 321, 3071, doi:10.1016/j.jmmm.2009.05.003

${ }^{16}$ E. Chason, P.R. Guduru, Tutorial: Understanding residual stress in polycrystalline thin films through real-time measurements and physical models, J. Appl. Phys., 2016, 119, 191101, 10.1063/1.4949263.

${ }^{17}$ C. Aroca, M. C. Sánchez, I. Tanarro, P. Sánchez, E. López, M. Vázquez, Induced anisotropies in ferromagnetic amorphous ribbons locally annealed by laser, Phys. Rev. B, 1990, 42, 8086, doi: 10.1103/PhysRevB.42.8086

${ }^{18}$ J. Sort, L.F. Bonavina, A. Varea, C. Souza, W.J. Botta, C.S. Kiminami, C. Bolfarini, S. Suriñach, M.D. Baro', J. Nogués, Out-of-Plane Magnetic Patterning Based on Indentation-Induced Nanocrystallization of a Metallic Glass, Small, 2010, 6, 1543, doi: 10.1002/smll.201000510

${ }^{19}$ G. Dai, Q. Zhan, Y. Liu, H. Yang, X. Zhang, B. Chen, R-W Li, Mechanically tunable magnetic properties of $\mathrm{Fe}_{81} \mathrm{Ga}_{19}$ films grown on flexible substrates, Appl. Phys. Lett., 2012, 100, 122407, doi:10.1063/1.3696887

${ }^{20}$ Y. Chen, Y. Mei, R. Kaltofen, J.I. Mönch, J. Schumann, J. Freudenberger, H-J. Klauß, O.G. Schmidt, Towards Flexible Magnetoelectronics: Buffer-Enhanced and Mechanically Tunable GMR 
of $\mathrm{Co} / \mathrm{Cu}$ Multilayers on Plastic Substrates, Adv. Mater., 2008, 20, 3224, doi: 10.1002/adma.200800230

${ }^{21}$ J.I. Martín, J. Nogués, K. Liu, J.L. Vicent, I.K. Schuller, Ordered magnetic nanostructures: fabrication and properties, J. Magn. Magn. Mater., 2003, 256, 449, doi: 10.1016/S03048853(02)00898-3

22 C. A. Ross, M. Hwang, M. Shima, J. Y. Cheng, M. Farhoud, T. A. Savas, Henry I. Smith, W. Schwarzacher, F. M. Ross, M. Redjdal, F.B. Humphrey, Micromagnetic behavior of electrodeposited cylinder arrays, Phys. Rev. B, 2002, 65, 144417, doi: 10.1103/PhysRevB.65.144417

${ }^{23}$ R.P. Cowburn, A.O. Adeyeye, J. A. C. Bland, Magnetic domain formation in lithographically defined antidot permalloy arrays, Appl. Phys. Lett., 1997, 70, 2309, doi: 10.1063/1.118845

${ }^{24}$ K.Yu.Guslienko, Magnetic anisotropy in two-dimensional dot arrays induced by magnetostatic interdot coupling, Physics Letters A, 2001, 278, 293, doi: 10.1016/S0375-9601(00)00793-3

${ }^{25}$ C.C. Wang, A.O. Adeyeye, N. Singh, Magnetic antidot nanostructures: effect of lattice geometry, Nanotechnology, 2006, 17, 1629, doi:10.1088/0957-4484/17/6/015

${ }^{26}$ C. Bran, P. Gawronski, I. Lucas, R. P. del Real, P. Strichovanec, A. Asenjo, M. Vazquez, O. Chubykalo-Fesenko, Magnetic hardening and domain structure in $\mathrm{Co} / \mathrm{Pt}$ antidots with perpendicular anisotropy, J. Phys. D: Appl. Phys., 2017, 50, 065003, doi:10.1088/1361-6463/aa4ee3

${ }^{27}$ F. Spizzo, E. Bonfiglioli, M. Tamisari, A. Gerardino, G. Barucca, A. Notargiacomo, F. Chinni, L. Del Bianco, Magnetic exchange coupling in $\mathrm{IrMn} / \mathrm{NiFe}$ nanostructures: From the continuous film to dot arrays, Phys. Rev. B., 2015, 91, 064410, doi: 10.1103/PhysRevB.91.064410.

${ }^{28}$ F. Spizzo, M. Tamisari, F. Chinni, E. Bonfiglioli, A. Gerardino, G. Barucca, D. Bisero, S. Fin, L. Del Bianco, Exchange bias properties of $140 \mathrm{~nm}$-sized dipolarly interacting circular dots with ultrafine IrMn and NiFe layers, J. Magn. Magn. Mater., 2016, 400, 242, doi:10.1016/j.jmmm.2015.08.001

${ }^{29}$ G. Gubbiotti, S. Tacchi, M. Madami, G. Carlotti, A.O. Adeyeye, M. Kostylev, Brillouin light scattering studies of planar metallic magnonic crystals, J. Phys. D: Appl. Phys., 2010, 43, 264003, doi:10.1088/0022-3727/43/26/264003

30 M. Coïsson, F. Celegato, G. Barrera, G. Conta, A. Magni, P. Tiberto, Bi-Component nanostructured arrays of Co dots embedded in $\mathrm{Ni}_{80} \mathrm{Fe}_{20}$ antidot matrix: synthesis by self-assembling of polystyrene nanospheres and magnetic properties, Nanomaterials, 2017, 7, 232, doi:10.3390/nano7090232

${ }^{31}$ B. Toal, M. McMillen, A. Murphy, W. Hendren, M. Arredondo, R. Pollard, Optical and magnetooptical properties of gold core cobalt shell magnetoplasmonic nanowire arrays, Nanoscale, 2014, 6 , 12905, doi:10.1039/c4nr03792h

32 S. David, C. Polonschii, C. Luculescu, M. Gheorghiu, S. Gáspár, E. Gheorghiu, Magnetoplasmonic biosensor with enhanced analytical response and stability, Biosensors and Bioelectronics, 2015, 63, 525, doi:10.1016/j.bios.2014.08.004 
${ }^{33}$ F. Chinni, F. Spizzo, F. Montoncello, V. Mattarello, C. Maurizio, G. Mattei, L. Del Bianco, Magnetic hysteresis in nanocomposite films consisting of a ferromagnetic AuCo alloy and ultrafine Co particles, Materials, 2017, 10, 717, doi:10.3390/ma10070717

${ }^{34}$ C. Maurizio, N. Michieli, B. Kalinic, V. Mattarello, V. Bello, F. Wilhelm, K. Ollefs, G. Mattei, Local structure and X-ray magnetic circular dichroism of Au in Au-Co nanoalloys, Applied Surface Science, 2018, 433, 596, doi:10.1016/j.apsusc.2017.10.067

${ }^{35}$ L. Li, S.H. Chai, A. Binder, S. Brown, S-Z. Yang, S. Dai, Synthesis of MCF-supported AuCo nanoparticle catalysts and the catalytic performance for the $\mathrm{CO}$ oxidation reaction, $R S C A d v$., 2015, 5, 100212, doi:10.1039/c5ra11487j

${ }^{36}$ J. C. Hulteen, D. A. Treichel, M. T. Smith, M. L. Duval, T. R. Jensen, and R. P. Van Duyne, Nanosphere Lithography: Size-Tunable Silver Nanoparticle and Surface Cluster Arrays, J. Phys. Chem. B, 1999, 103, 3854, doi: 10.1021/jp9904771

${ }^{37}$ P. Colson, C. Henrist, R. Cloots, Nanosphere lithography: a powerful method for the controlled manufacturing of nanomaterials, Journal of Nanomaterials, 2013, 2013, 948510, doi:10.1155/2013/948510

${ }^{38}$ V. Russo, N. Michieli, T. Cesca, C. Scian, D. Silvestri, M. Morpurgo, G. Mattei, Gold-silver alloy semi-nanoshell arrays for label-free plasmonic biosensors, Nanoscale, 2017, 9, 10117, doi: $10.1039 / \mathrm{c} 7 \mathrm{nr} 01982 \mathrm{c}$

${ }^{39}$ T. Cesca, N. Michieli, B. Kalinic, A. Sánchez-Espinoza, M. Rattin, V. Russo, V. Mattarello, C. Scian, P. Mazzoldi, G. Mattei, Nonlinear absorption tuning by composition control in bimetallic plasmonic nanoprism arrays, Nanoscale, 2015, 7, 12411, doi: 10.1039/C5NR01715G

${ }^{40}$ H. Sánchez-Esquivel, K. Y. Raygoza-Sánchez, R. Rangel-Rojo, E. Gemo, N. Michieli, B. Kalinic, J. A. Reyes-Esqueda, T. Cesca, G. Mattei, Spectral dependence of nonlinear absorption in ordered silver metallic nanoprism arrays, Scientific Reports, 2017, 7, 5307, doi:10.1038/s41598017-04814-2

${ }^{41}$ A. Vansteenkiste, J. Leliaert, M. Dvornik, M. Helsen, F. Garcia-Sanchez, B. Van Waeyenberge, The design and verification of mumax3, AIP Advances, 2014, 4, 107133, doi: 10.1063/1.4899186

${ }^{42}$ V. Mattarello, Au-Co Thin Films and Nanostructures for MagnetoPlasmonics, PhD Thesis Department of Chemical Sciences, University of Padova, Italy, 2016.

43 B. Presa, R. Matarranz, M.C. Contreras, J. F. Calleja, L.E. Fernandez-Outon, K.O'Grady, Magnetic Properties of Nanocrystalline Co Thin Films Grown on Glass, IEEE Trans. Magn., 2008, 44, 2788, doi: 10.1109/TMAG.2008.2001506

${ }^{44}$ L.M. Álvarez-Prado, J.M. Alameda, Micromagnetism of nanowires with low out-of planeanisotropy, Physica B, 2004, 343, 241, doi: 10.1016/j.physb.2003.08.101 
${ }^{45}$ M. Coïsson, F. Celegato, E. Olivetti, P. Tiberto, F. Vinai, M. Baricco, Stripe domains and spin reorientation transition in $\mathrm{Fe}_{78} \mathrm{~B}_{13} \mathrm{Si}_{9}$ thin films produced by rf sputtering, J. Appl. Phys., 2008, 104, 033902, doi: 10.1063/1.2960454

${ }^{46}$ A. V. Svalov, G. V. Kurlyandskaya, H. Hammer, P. A. Savin, and O. I. Tutynina, Modification of the "transcritical" state in Ni Fe Cu Mo films produced by RF sputtering, Tech. Phys., 2004, 49, 868, doi: $10.1134 / 1.1778860$

${ }^{47}$ A. Hubert and S. Schäfer, Magnetic Domains, Springer-Verlag, Berlin Heidelberg, 1998.

${ }^{48}$ G.G. Stoney, The Tension of Metallic Films Deposited by Electrolysis, Proc. R. Soc. London, 1909, A82, 172, doi:10.1098/rspa.1909.0021.

${ }^{49}$ X. Feng, Y. Huang, A.J. Rosakis, On the Stoney formula for a thin film/substrate system with nonuniform substrate thickness, J. Appl. Mech., 2007, 74, 1276, doi: 10.1115/1.2745392.

${ }^{50}$ N. Saito, H. Fujiwara, Y. Sugita, A new type of magnetic domain structure in negative magnetostriction Ni-Fe films, J. Phys. Soc. Japan, 1964, 19, 1116, doi: 10.1143/JPSJ.19.1116

51 L.M. Alvarez-Prado, G.T. Pérez, R. Morales, F.H. Salas, J. M. Alameda, Perpendicular anisotropy detected by transversely biased initial susceptibility via the magneto-optic Kerr effect in $\mathrm{Fe}_{x} \mathrm{Si}_{1-x}$ thin films and $\mathrm{Fe}_{x} \mathrm{Si}_{1-x} / \mathrm{Si}$ multilayers: Theory and experiment, Phys. Rev. B, 1997, 56, 3306, doi:10.1103/PhysRevB.56.3306

${ }^{52}$ M. Hehn, S. Padovani, K. Ounadjela, J. P. Bucher, Nanoscale magnetic domain structures in epitaxial cobalt films, Phys. Rev. B, 1996, 54, 3428, doi: 10.1103/PhysRevB.54.3428

${ }^{53}$ M. Barturen, B. Rache Salles, P. Schio, J. Milano, A. Butera, S. Bustingorry, C. Ramos, A. J. A. de Oliveira, M. Eddrief, E. Lacaze, F. Gendron, V. H. Etgens, M. Marangolo, Crossover to striped magnetic domains in $\mathrm{Fe}_{1-\mathrm{x}} \mathrm{Ga}_{\mathrm{x}}$ magnetostrictive thin films, Appl. Phys. Lett., 2012, 101, 092404, doi: $10.1063 / 1.4748122$

${ }^{54}$ C. Banerjee, P. Gruszecki, J.W. Klos, O. Hellwig, M. Krawczyk, A. Barman, Magnonic band structure in a $\mathrm{Co} / \mathrm{Pd}$ stripe domain system investigated by Brillouin light scattering and micromagnetic simulations, Phys. Rev. B, 2017, 96, 024421, doi: 10.1103/PhysRevB.96.024421

55 A. Ovadia, I.B. Puchalska, J.P. Jakubovics, Stripe domains in obliquely deposited Co-Au films, J. Magn. Magn. Mater., 1986, 54-57, 851, doi:10.1016/0304-8853(86)90282-9

${ }^{56}$ E. E. Huber Jr., D. O. Smith, Properties of permalloy films having a magnetoelastic easy axis normal to the film, J. Appl. Phys., 1959, 30, 267S, doi: 10.1063/1.2185923

${ }^{57}$ J. Sort, H. Glaczynska, U. Ebels, B. Dieny, M. Giersig, J. Rybczynski, Exchange bias effects in submicron antiferromagnetic-ferromagnetic dots prepared by nanosphere lithography, J. Appl. Phys., 2004, 95, 7516, doi:10.1063/1.1682991

${ }^{58}$ W. Ingram, Y. He, K. Stone, W.M Dennis, D. Ye, Y. Zhao, Tuning the plasmonic properties of silver nanopatterns fabricated by shadow nanosphere lithography, Nanotechnology, 2016, 27, 385301, doi:10.1088/0957-4484/27/38/385301 
59 A. Kosiorek, W. Kandulski, P. Chudzinski, K. Kempa, M. Giersig, Shadow nanosphere lithography: simulation and experiment, Nano Letters, 2004, 2, 1359, doi: 10.1021/n1049361t

${ }^{60}$ A. Manzin, O Bottauscio, Micromagnetic modelling of the anisotropy properties of permalloy antidot arrays with hexagonal symmetry, J. Phys. D: Appl. Phys., 2012, 45, 095001, doi:10.1088/0022-3727/45/9/095001

${ }^{61}$ A. Manzin, G. Barrera, F. Celegato, M. Coïsson, P. Tiberto, Influence of lattice defects on the ferromagnetic resonance behaviour of 2D magnonic crystals, Sci. Rep., 2016, 6, 22004, doi: $10.1038 /$ srep22004

${ }^{62}$ M. Jaafar, D. Navas, A. Asenjo, M. Vázquez, M. Hernández-Vélez, J.M. García-Martín, Magnetic domain structure of nanohole arrays in Ni films, J. Appl. Phys., 2007, 101, 09F513, doi: $10.1063 / 1.2711613$

${ }^{63}$ A. Schwarz, R. Wiesendanger, Magnetic sensitive force microscopy, nanotoday, 2008, 3, 28, 10.1016/S1748-0132(08)70013-6 


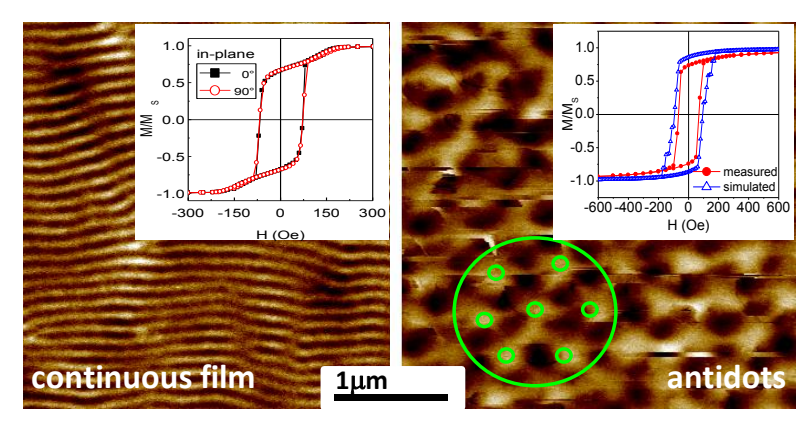

The nanopatterning of CoAu films enables a local modification of the shape factor and a tuning of the interplay of magnetic anisotropies. 